\title{
Análise da Depressão, dos Fatores de Risco para Sintomas Depressivos e do Uso de Antidepressivos entre Acadêmicos do Curso de Medicina da Universidade Estadual de Ponta Grossa
}

An Analysis of Depression, the Risk Factors for Depressive Symptoms, and the Use of Antidepressants among Medical Students at Ponta Grossa State University

\section{PALAVRAS-CHAVE}

- Antidepressivos.

- Depressão.

- Estudantes de Medicina.

- Transtorno Depressivo.

- Transtornos do Humor.

- Prevalência.

Recebido em: 10/10/2016

Aprovado Em: 21/10/2016

\section{RESUMO}

Introdução: A depressão entre estudantes de Medicina tem sido uma condição prevalente, porém as pesquisas têm sido metodologicamente insuficientes quanto à análise dos fatores de risco envolvidos e ao tratamento dessa população. Objetivos: Determinar a prevalência de sintomas depressivos e de seus fatores de risco, assim como do uso de antidepressivos na amostra analisada. Método: Para o screening de sintomas depressivos, foi aplicado o Inventário de Depressão de Beck (BDI), e para a adesão medicamentosa, o teste de Morisky-Green-Levine. O teste exato de Fisher unicaudado foi utilizado para variáveis qualitativas e, para as quantitativas, o teste one-way Anova com análise post-hoc pelo teste de Tukey-Kramer. As diferenças foram consideradas significativas quando $p<0,05$. Resultados: Mostraram-se estatisticamente significativas as associações entre sintomas depressivos e frequência de atividades de lazer, estresse, satisfação com o desempenho acadêmico e falta de apoio emocional. Não mostraram associação com sintomas depressivos as seguintes variáveis: sexo, viver sozinho, parceiro fixo, álcool, tabagismo, drogas ilícitas e série do curso. A satisfação com o desempenho acadêmico e o alto grau de estresse não apresentaram significância estatística ao serem relacionados com as séries do curso. Conclusão: As prevalências de sintomas depressivos nos acadêmicos de Medicina da UEPG e da utilização de medicamentos antidepressivos vão ao encontro dos dados referentes a acadêmicos de Medicina de outras instituições brasileiras e internacionais. Apresentam-se como fatores de risco para os transtornos depressivos a frequência das atividades de lazer, o estresse, a satisfação com o desempenho acadêmico e a falta de apoio emocional no ambiente acadêmico. A prevalência de depressão a partir da quarta série tendeu a aumentar, e apenas a sexta série diferiu de modo estatisticamente significativo em relação às demais quanto às médias do escore BDI, o que sugere a hipótese de que o final do curso de Medicina é o período que apresenta maior quantidade de fatores estressores e depressivos para o acadêmico. 


\author{
KEYWORDS \\ - Antidepressants. \\ - Depression. \\ - Medical Students. \\ - Depressive Disorder. \\ - Mood Disorders \\ - Prevalence.
}

\begin{abstract}
Introduction: Depression among medical students is particularly prevalent, however studies on risk factors and treatment for this group have proved to be methodologically insufficient as the results were not consistent. Aims: To determine the prevalence of depressive symptoms, their risk factors, and the use of antidepressants among medical students. Methods: Participants answered the Beck's Inventory (BDI) for detecting depressive symptoms and the Morisky-Green-Levine test to evaluate medication adherence. A one-tailed Fisher's exact test was performed on qualitative variables, with a one-way Anova test performed on quantitative variables with post-hoc analysis by means of Tukey-Kramer's test. Differences were considered to be statistically significant when $p<0.05$. Results: Associations between depressive disorders and the following factors were considered statistically significant: frequency of leisure activities, stress, satisfaction with academic performance, and lack of emotional support. Gender, living alone, steady partner, use of alcohol, smoking tobacco, use of illicit drugs, and Medical school's grade did not show association with depressive disorders. Satisfaction with academic performance and high level of stress were not statistically significant when they were correlated to Medical school's grade. Conclusion: The prevalence of depression and also use of antidepressants is similar to the results recorded by other studies on medical students performed by Brazilian and international institutions. The following items were considered to be risk factors on the development of depressive disorders: frequency of leisure activities, stress, satisfaction with academic performance, and lack of emotional support in the academic environment. From the fourth grade to the sixth grade, the prevalence of depression seemed to increase and just the sixth grade was statistically significant when compared to other grades through averages of BDI score. It suggests the hypothesis that the end of Medical course is the period of time where there are more stressful and depressive factors for the student.
\end{abstract}

\section{INTRODUÇÃO}

A depressão é considerada a condição mais incapacitante e penosa que existe ${ }^{1}$. É uma doença complexa, que pode levar a complicações que atingem inúmeras esferas da vida de um indivíduo. No cenário do estudante de Medicina, isto inclui o desempenho acadêmico e a relação médico-paciente.

Essa patologia é particularmente preocupante devido ao risco inerente de suicídio associado, que acomete cerca de $10 \%$ a $15 \%$ dos indivíduos com depressão grave 2 . Por isso, é importante reconhecer e tratar os sintomas depressivos em tempo hábil, devendo-se avaliar e acompanhar a resposta ao tratamento, bem como as decisões tomadas em relação ao mesmo.

A prevalência na população geral para transtornos depressivos tem alcançado números entre $4 \%$ e $10 \%$, sendo observada maior incidência em mulheres ${ }^{3}$. A frequência dos quadros depressivos é maior na população jovem estudantil do que na população geral. Alguns estudos apontam que de $15 \%$ a 29\% dos estudantes universitários apresentam algum tipo de transtorno psiquiátrico durante sua vida acadêmica e sugerem que $28 \%$ dos brasileiros entre 18 e 24 anos apresentam sintomas depressivos ${ }^{1-5}$.

A prevalência de sintomas depressivos entre estudantes de Medicina é uma constante em nível mundial, e seus valores possuem um grande intervalo de variação, entre 13,9\% e 79\% \%- $^{6-}$ ${ }^{14}$.A depressão pode causar grande sofrimento psíquico e levar a prejuízos no desempenho acadêmico e nos relacionamentos sociais. A provável existência de diferentes agentes estressores ao longo do curso de Medicina, que podem levar ao desenvolvimento de sintomas depressivos - como perda da liberdade pessoal, alto nível de exigência e de conteúdo, sentimento de desumanização, falta de tempo para o lazer, forte competição entre os colegas e o próprio contato com pacientes, com a dor e a doença -, parece depender do período e da série em que o aluno se encontra ${ }^{11,12,15,16}$. Por isso, procurou-se estudar os fatores de risco relacionados com a depressão entre estudantes de Medicina. Poucas horas de lazer e estresse parecem ser importantes fatores de risco para depressão nesta população $0^{8,17,18,19}$. Entretanto, a prevalência de tratamento dos sintomas depressivos parece ser baixa ${ }^{20}$.

É evidente o aparecimento de sintomas depressivos nos acadêmicos de Medicina ao longo da sua formação, os quais, por conviverem precocemente com a dor humana, podem ter maior probabilidade de desenvolver quadros depressivos. Como se trata de uma doença biológica relacionada com a atividade das monoaminas, o transtorno depressivo é passível de tratamento medicamentoso $0^{4,21}$. 
Além da teoria monoaminérgica da etiologia da depressão, amplamente difundida, considera-se que a depressão é uma síndrome heterogênea e multifatorial, uma vez que condições genéticas, ambientais e comportamentais regulam a sua expressão. Por ser uma síndrome complexa, torna-se difícil a identificação da fisiopatologia subjacente, o que acaba por complicar ainda mais seu diagnóstico e tratamento ${ }^{22}$.

Esta pesquisa consiste num estudo descritivo do tipo transversal cujo objetivo foi detectar, entre estudantes de Medicina, a prevalência de sintomas depressivos e os fatores correlacionados, assim como a prevalência e adesão ao uso de medicamentos antidepressivos.

\section{MÉTODOS}

Para a realização do estudo, foram aplicados questionários aos acadêmicos do curso de Medicina da Universidade Estadual de Ponta Grossa (UEPG) da primeira à sexta série. Utilizaram-se três tipos de questionários: um com o levantamento das questões socioeconômicas e demográficas dos acadêmicos ${ }^{8}$, outro para o screening de sintomas depressivos (Inventário de Depressão de Beck-BDI) $)^{8,23-25}$ e o último para obter informações sobre o uso de medicamentos antidepressivos (adaptado pela pesquisadora) e sobre a adesão medicamentosa (teste de Morisky-Green-Levine) $)^{26-28}$. Os dados foram coletados entre setembro de 2014 e fevereiro de 2015, períodos que abrangem o mesmo ano letivo, uma vez que este se inicia no mês de agosto nesta universidade.

Dos 239 acadêmicos matriculados no curso de Medicina no período do estudo, 199 participaram da pesquisa. Foram selecionados e incluídos todos os acadêmicos matriculados no curso de Medicina. Foram excluídos aqueles que se negaram a participar do estudo, que estavam em mobilidade internacional ou que não estavam presentes nos momentos de aplicação dos questionários. Por não estarem presentes nos momentos da coleta de dados ou por terem recusado a participação, 40 acadêmicos foram excluídos da amostra.

O curso de Medicina da Universidade Estadual de Ponta Grossa (UEPG) estrutura-se basicamente em dois períodos: ciclo básico (primeira à terceira série) e ciclo clínico (quarta série e o internato médico - quinta e sextas séries, período de maior contato com os pacientes e de prática clínica).

Os estudantes foram analisados quanto às seguintes variáveis: série do curso (primeira à sexta série), idade, sexo, viver sozinho, nível de estresse (nulo, baixo, médio ou alto), horas de lazer por semana (0 a 2; 2 a 14; mais de 14), parceiro fixo, uso de álcool e de drogas ilícitas, tabagismo, satisfação com o desempenho acadêmico e com a escolha do curso e falta de apoio emocional. A presença e os tipos de sintomas depres- sivos também foram pesquisados, assim como o uso de antidepressivos no momento da pesquisa e previamente, a classe de antidepressivos e o tempo de uso dos mesmos. O grau de adesão medicamentosa foi estratificado em baixo (3-4 pontos), mediano (1-2 pontos) e alto (nenhum ponto).

Foram considerados portadores de sintomas depressivos os acadêmicos que apresentaram escore $\geq 11$ pontos no BDI, por ser este o ponto de corte considerado o melhor limiar para detectar depressão, alcançando sensibilidade de 70\% e especificidade de $87 \%{ }^{25}$. O BDI consiste em 21 questões relacionadas com os transtornos depressivos, que geram um escore que varia de 0 a 63 pontos. Este inventário avalia aspectos relacionados com tristeza, pessimismo, sensação de fracasso, falta de satisfação, sensação de culpa e de punição, autodepreciação, autoacusações, ideias suicidas, crises de choro, irritabilidade, retração social, indecisão, distorção da imagem corporal, inibição para o trabalho, distúrbio do sono, fadiga, perda de apetite e de peso, preocupação somática e diminuição da libido ${ }^{29,30}$. Com base nas respostas, somam-se os pontos e se obtém a Escala de Depressão de Beck (EDB), que pode ser estratificada da seguinte maneira: 11 a 16 pontos para distúrbio moderado do humor; 17 a 20 pontos para depressão clínica borderline; 21 a 30 pontos para depressão moderada; 31 a 40 pontos para depressão severa; e escores superiores a 40 pontos para depressão extrema ${ }^{30}$.

Todos os acadêmicos participantes da pesquisa tiveram acesso ao Termo de Consentimento Livre e Esclarecido (TCLE). O estudo foi realizado mediante autorização do Comitê de Ética em Pesquisa (Coep) da Universidade Estadual de Ponta Grossa (UEPG), aprovado em 26/09/2014, Parecer $n^{\circ}$ 808.102, conforme a Resolução do Conselho Nacional de Saúde 196/6 para pesquisa envolvendo seres humanos.

Os dados obtidos foram testados quanto à normalidade de distribuição por meio das médias e desvios padrão. Para as variáveis quantitativas, a comparação das médias entre os grupos (distribuições normais) foi determinada utilizando-se o teste paramétrico one-way Anova com análise post-hoc por meio do teste de Tukey-Kramer.

Esta foi uma investigação exploratória que desejava estabelecer se os sintomas depressivos estariam ou não relacionados com determinada série do curso de Medicina a fim de gerar hipóteses para estudos futuros. Comparações foram realizadas entre as seis séries do curso, entre os dois períodos estabelecidos (básico: primeira à terceira série, clínico: quarta à sexta série) e entre as séries do ciclo clínico (quarta à sexta série). Para a comparação das variáveis qualitativas foi aplicado o teste exato de Fisher unicaudado. As diferenças foram consideradas significativas quando $p<0,05^{31}$. O software MedCalc ${ }^{\circledR}$ 14.8.1 foi utilizado para os cálculos estatísticos. 


\section{RESULTADOS}

A maioria dos estudantes de Medicina participantes do estudo apresentava idades entre 18 e 25 anos. Mais da metade dos estudantes tinha parceiro fixo (cônjuge, relacionamento sério), pertencia ao sexo masculino e utilizava bebidas alcoólicas. Chamou a atenção o fato de a prevalência de alto nível de estresse ter sido mais elevada do que a dos níveis de estresse nulo, leve e médio (Tabelas 1, 2 e 3).

\begin{tabular}{|c|c|c|c|c|c|c|c|}
\hline \multicolumn{8}{|c|}{ TABela 1} \\
\hline & 1é Série & 2a Série & $3^{\text {a }}$ Série & $4^{\text {a }}$ Série & 5a Série & 6 a $^{\text {Série }}$ & Total \\
\hline & $32(16.08 \%)$ & $42(21.06)$ & $20(10.05 \%)$ & $34(17.09 \%)$ & $41(20.6 \%)$ & $30(15.08 \%)$ & $199(100 \%)$ \\
\hline \multicolumn{8}{|l|}{ Nível de estresse } \\
\hline Nulo & 0 & 0 & 0 & 0 & $1(2.44 \%)$ & 0 & $1(0.5 \%)$ \\
\hline Baixo & $3(9.38 \%)$ & $3(7.14 \%)$ & 0 & 0 & $2(4.88 \%)$ & $2(6.67 \%)$ & $10(5.03 \%)$ \\
\hline Médio & $8(25 \%)$ & $10(23.81 \%)$ & $5(25 \%)$ & $9(26.47 \%)$ & $13(31.71 \%)$ & $10(33.33 \%)$ & $55(27.64 \%)$ \\
\hline Alto & $21(65.63 \%)$ & $29(69.05 \%)$ & $15(75 \%)$ & $25(73.53 \%)$ & $25(60.98 \%)$ & $18(60 \%)$ & $133(66.83 \%)$ \\
\hline \multicolumn{8}{|l|}{ Satisfação } \\
\hline Desempenho acadêmico & $10(31.25 \%)$ & $14(33.33 \%)$ & $9(45 \%)$ & $13(38.24 \%)$ & $22(53.66 \%)$ & $18(60 \%)$ & $86(43.22 \%)$ \\
\hline Escolha do curso & $25(78.13 \%)$ & $37(88.1 \%)$ & $19(95 \%)$ & $31(91.18 \%)$ & $39(95.12 \%)$ & $28(93.33 \%)$ & $179(89.95 \%)$ \\
\hline
\end{tabular}

\section{TABela 2}

Dados subjetivos pessoais dos participantes do estudo e sua variação conforme a série do curso

\begin{tabular}{ccccc} 
& \multicolumn{2}{c}{ Satisfeito com o desempenho acadêmico } & \multicolumn{2}{c}{ Considera-se com alto nível de estresse? } \\
Série & Sim & Não & Sim & $11(34.37 \%)$ \\
\hline 1 & $10(31.25 \%)$ & $22(68.75 \%)$ & $21(65.63 \%)$ & $13(30.95 \%)$ \\
\hline 2 & $14(33.33 \%)$ & $28(66.67 \%)$ & $29(69.05 \%)$ & $5(25 \%)$ \\
\hline 3 & $9(45 \%)$ & $11(55 \%)$ & $15(75 \%)$ & $9(26.47 \%)$ \\
\hline 4 & $13(38.24 \%)$ & $21(61.76 \%)$ & $25(73.53 \%)$ & $16(39.02 \%)$ \\
\hline 5 & $22(53.66 \%)$ & $19(46.34 \%)$ & $25(60.98 \%)$ & $12(40 \%)$ \\
\end{tabular}

* Teste $x 2$ unicaudado

\begin{tabular}{|c|c|c|c|c|c|c|c|}
\hline \multicolumn{8}{|c|}{ TABELA 3} \\
\hline \multicolumn{8}{|c|}{ Fatores socioeconômicos e demográficos dos acadêmicos pesquisados } \\
\hline & 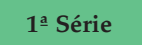 & $2^{\text {a }}$ Série & 3a Série & $4^{\text {a }}$ Série & $5^{\text {a }}$ Série & 6 ${ }^{\text {a }}$ Série & TOTAL \\
\hline & $32(16.08 \%)$ & $42(21.06)$ & $20(10.05 \%)$ & $34(17.09 \%)$ & $41(20.6 \%)$ & $30(15.08 \%)$ & $199(100 \%)$ \\
\hline \multicolumn{8}{|l|}{ Idade (anos) } \\
\hline $18-25$ & $32(100 \%)$ & $39(92.86 \%)$ & $19(95 \%)$ & $30(88.24 \%)$ & $29(70.73 \%)$ & $17(56.67 \%)$ & $166(83.42 \%)$ \\
\hline $25-30$ & 0 & $2(4.76 \%)$ & 0 & $2(5.88 \%)$ & $2(4.88 \%)$ & $11(36.67 \%)$ & 17 (8.54\%) \\
\hline$>30$ & 0 & $1(2.38 \%)$ & $1(5 \%)$ & $2(5.88 \%)$ & $10(24.39 \%)$ & $2(6.67 \%)$ & $16(8.04 \%)$ \\
\hline \multicolumn{8}{|l|}{ Sexo } \\
\hline Masculino & $17(53.13 \%)$ & $25(59.52 \%)$ & $13(65 \%)$ & $18(52.94 \%)$ & $23(56.1 \%)$ & $12(40 \%)$ & $108(54.27 \%)$ \\
\hline Feminino & $15(46.88 \%)$ & $17(40.48 \%)$ & $7(35 \%)$ & $16(47.05 \%)$ & $18(43.9 \%)$ & $18(60 \%)$ & $91(45.73 \%)$ \\
\hline Vive sozinho & $9(28.13 \%)$ & $17(40.48 \%)$ & $8(40 \%)$ & $19(55.88 \%)$ & $14(34.15 \%)$ & $10(33.33 \%)$ & $77(38.69 \%)$ \\
\hline \multicolumn{8}{|l|}{ Horas de lazer/semana } \\
\hline $0-2$ & $5(15.63 \%)$ & $14(33.34 \%)$ & $2(10 \%)$ & $6(17.65 \%)$ & $6(14.63 \%)$ & $6(20 \%)$ & $39(19.6 \%)$ \\
\hline 2 a 14 & $23(71.88 \%)$ & $24(57.14 \%)$ & $10(50 \%)$ & $23(67.65 \%)$ & $31(75.61 \%)$ & $16(53.33 \%)$ & $127(63.82 \%)$ \\
\hline$>14$ & $4(12.5 \%)$ & $4(9.52 \%)$ & $8(40 \%)$ & $5(14.71 \%)$ & $4(9.76 \%)$ & $8(26.67 \%)$ & $33(16.59 \%)$ \\
\hline Parceiro fixo & $14(43.75 \%)$ & $19(45.24 \%)$ & $11(55 \%)$ & $24(70.59 \%)$ & $28(68.29 \%)$ & $19(63.33 \%)$ & $115(57.79 \%)$ \\
\hline Álcool & $15(46.88 \%)$ & $17(40.48 \%)$ & $11(55 \%)$ & $19(55.88 \%)$ & $28(68.29 \%)$ & $14(46.67 \%)$ & $104(52.26 \%)$ \\
\hline Tabagismo & $2(6.25 \%)$ & 0 & $2(10 \%)$ & $3(8.82 \%)$ & $4(9.76 \%)$ & $2(6.67 \%)$ & $13(6.53 \%)$ \\
\hline Drogas ilícitas & $8(25 \%)$ & $3(7.14 \%)$ & $6(30 \%)$ & 12 (35.29\%) & $15(36.59 \%)$ & $7(23.33 \%)$ & $51(25.63 \%)$ \\
\hline Curso superior prévio & $7(21.88 \%)$ & $10(23.81 \%)$ & $4(20 \%)$ & $10(29.41 \%)$ & $9(21.95 \%)$ & $8(26.67 \%)$ & $48(24.12 \%)$ \\
\hline Falta de apoio emocional & $20(62.5 \%)$ & $26(61.9 \%)$ & $11(55 \%)$ & $19(55.88 \%)$ & $19(55.88 \%)$ & $19(63.33 \%)$ & $114(57.29 \%)$ \\
\hline
\end{tabular}




\begin{tabular}{|c|c|c|c|c|c|c|c|}
\hline \multicolumn{8}{|c|}{$\begin{array}{l}\text { TА ВЕLA } 4 \\
\text { essão e de uso de antidepressivos }\end{array}$} \\
\hline & $1^{\text {a }}$ Série & 2a Série & 3a Série & $4^{\text {a }}$ Série & $5^{a}$ Série & 6 Série & Total \\
\hline & $32(16.08 \%)$ & $42(21.06)$ & $20(10.05 \%)$ & $34(17.09 \%)$ & $41(20.6 \%)$ & $30(15.08 \%)$ & $199(100 \%)$ \\
\hline Prevalência de depressão & $17(53.13 \%)$ & $21(50 \%)$ & $10(50 \%)$ & $12(35.29 \%)$ & $12(29.27 \%)$ & $16(53.33 \%)$ & $88(44.22 \%)$ \\
\hline Escore de Beck & $12.22(7.13)$ & $11.88(7.21)$ & $9.95(6.67)$ & $9.5(6.76)$ & $8.28(5.75)$ & $9.73(5.39)$ & $10.26(6.49)$ \\
\hline \multicolumn{8}{|l|}{ Tipo de transtorno depressivo } \\
\hline Normal & $15(46.88 \%)$ & $21(50 \%)$ & $10(50 \%)$ & $22(64.71 \%)$ & $29(70.73 \%)$ & $14(46.67 \%)$ & $111(55.78 \%)$ \\
\hline Distúrbio moderado do humor & $9(28.13 \%)$ & $12(28.57 \%)$ & $8(40 \%)$ & $8(23.53 \%)$ & $8(19.51 \%)$ & $14(46.67 \%)$ & $59(29.65 \%)$ \\
\hline Depressão clínica borderline & $4(12.5 \%)$ & $6(14.29 \%)$ & 0 & $2(5.88 \%)$ & $3(7.32 \%)$ & $2(6.67 \%)$ & $17(8.54 \%)$ \\
\hline Depressão moderada & $4(12.5 \%)$ & $2(4.76 \%)$ & $2(10 \%)$ & $1(2.94 \%)$ & $1(2.44 \%)$ & 0 & $10(5.03 \%)$ \\
\hline Depressão severa & 0 & 0 & 0 & $1(2.94 \%)$ & 0 & 0 & $1(0.05 \%)$ \\
\hline Depressão extrema & 0 & $1(2.38 \%)$ & 0 & 0 & 0 & 0 & $1(0.05 \%)$ \\
\hline Uso atual de antidepressivo & $4(12.5 \%)$ & $8(19.04 \%)$ & $3(15 \%)$ & $10(29.41 \%)$ & $8(19.51 \%)$ & $3(10 \%)$ & $36(18.09 \%)$ \\
\hline Tempo (meses) de uso do mesmo & $4.33(2.05)$ & $16.6(21.62)$ & $5.17(4.42)$ & $41(48.93)$ & $28(30.06)$ & $26(12.33)$ & $28.18(20.18)$ \\
\hline Uso prévio de antidepressivo & $4(12.5 \%)$ & $2(4.76 \%)$ & $2(10 \%)$ & $6(17.65 \%)$ & $4(9.76 \%)$ & $4(13.33 \%)$ & $22(9.05 \%)$ \\
\hline Uso global de antidepressivo (atual e prévio) & $8(25 \%)$ & $10(23.8 \%)$ & $5(25 \%)$ & $16(47.09 \%)$ & $12(29.27 \%)$ & $7(23.33 \%)$ & $58(29.15 \%)$ \\
\hline \multicolumn{8}{|l|}{ Classe de antidepressivo } \\
\hline ADT & 0 & 0 & 0 & $1(29.41 \%)$ & 0 & 0 & $1(2.78 \%)$ \\
\hline ISRS & $2(6.25 \%)$ & $6(14.29 \%)$ & $3(15 \%)$ & $7(17.65 \%)$ & $8(19.51 \%)$ & $1(3 \%)$ & $27(75 \%)$ \\
\hline IRSN & $2(6.25 \%)$ & $2(4.76 \%)$ & 0 & $3(8.82 \%)$ & 0 & $1(3 \%)$ & $8(22.22 \%)$ \\
\hline Atípico & 0 & $1(2.38 \%)$ & $1(5 \%)$ & 0 & $1(2.44 \%)$ & $1(3 \%)$ & $4(11.11 \%)$ \\
\hline \multicolumn{8}{|l|}{ Adesão medicamentosa } \\
\hline Baixa & 0 & $2(20 \%)$ & 0 & $3(30 \%)$ & 0 & $1(33.33 \%)$ & $6(16.67 \%)$ \\
\hline Mediana & $2(50 \%)$ & $2(20 \%)$ & $2(66.67 \%)$ & $5(50 \%)$ & $5(62.5 \%)$ & $2(66.67 \%)$ & $18(50 \%)$ \\
\hline Alta & $2(50 \%)$ & $4(40 \%)$ & $1(33.33 \%)$ & $2(20 \%)$ & $3(37.5 \%)$ & 0 & $12(33.33 \%)$ \\
\hline
\end{tabular}

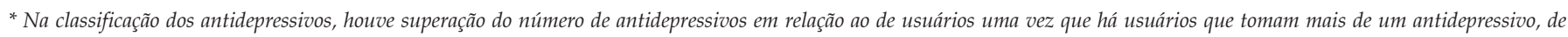
classe diferente

De acordo com os escores do BDI estabelecidos para este estudo, observou-se que menos da metade do total dos acadêmicos estudados apresentavam sintomas depressivos com ou sem tratamento (Tabela 4). Entre os portadores de sintomas depressivos, o tipo de transtorno depressivo mais frequente foi o distúrbio moderado do humor, seguido em ordem decrescente por depressão clínica borderline e depressão moderada. Depressão severa e depressão extrema apresentaram prevalências pequenas.

No momento da pesquisa, alguns alunos admitiram estar fazendo uso de terapia medicamentosa antidepressiva, porém esse número considera também aqueles que utilizaram previamente medicamentos antidepressivos. A classe mais utilizada foi a dos Inibidores Seletivos da Recaptação de Serotonina (ISRS). Os Inibidores dos Receptores da Serotonina-Noradrenalina (IRSN) foram a segunda classe de antidepressivos mais utilizada, seguida pelos antidepressivos atípicos e pelos antidepressivos tricíclicos (ADT). Entre os usuários de fármacos antidepressivos, a maioria apresentava algum tipo de transtorno depressivo mesmo com o tratamento $\mathrm{O}$ grau de adesão ao tratamento medicamentoso relatado foi considerado baixo para uma minoria dos pesquisados (Tabela 4).
Entre os acadêmicos pesquisados, grande parte se encontrava satisfeita com a escolha do curso. Mais da metade dos indivíduos pesquisados admitiram sentir falta de apoio emocional no ambiente acadêmico. Também se observou que as variáveis referentes a estresse e frequência de atividades de lazer apresentaram-se estatisticamente significativas no que diz respeito a fatores de risco para depressão. Não mostraram associação com sintomas depressivos as seguintes variáveis: sexo, viver sozinho, parceiro fixo, álcool, tabagismo, drogas ilícitas e série do curso (Tabelas 1, 2, 3 e 5).

A satisfação com o desempenho acadêmico e o alto grau de estresse não apresentaram significância estatística ao serem relacionados com as séries do curso (Tabela 2).

A análise comparativa entre as séries do ciclo clínico (quarta à sexta série) gerou um valor de $p$ estatisticamente significativo ao ser aplicado o teste exato de Fisher unicaudado (Tabela 5).

A comparação das médias dos escores do BDI entre as séries do curso, pelo teste Anova de via única, detectou que apenas a sexta série diferiu de modo estatisticamente significativo das demais turmas (Tabela 6). 


\begin{tabular}{|c|c|c|c|c|c|}
\hline \multicolumn{6}{|c|}{$\begin{array}{l}\text { TABELA } 5 \\
\text { são e fatores sociodemográficos e relativos ao curso, } \\
\text { rentes aos acadêmicos de Medicina da Universidade } \\
\text { período de setembro de } 2014 \text { a fevereiro de } 2015\end{array}$} \\
\hline & $\begin{array}{l}\text { Depressão } \\
\qquad \mathrm{n}=88\end{array}$ & $\begin{array}{l}\text { Não-depressão } \\
\qquad \mathbf{n = 1 1 1}\end{array}$ & $\mathbf{P}$ & OR & IC $95 \%$ \\
\hline \multicolumn{6}{|l|}{ Sexo } \\
\hline $\begin{array}{l}\text { Masculino } \\
\text { Feminino } \\
\text { Vive sozinho }\end{array}$ & $\begin{array}{l}47(53.41 \%) \\
41(46.59 \%) \\
30(34.09 \%)\end{array}$ & $\begin{array}{l}61(54.95 \%) \\
50(45.05 \%) \\
47(42.34 \%)\end{array}$ & $\begin{array}{l}p=0.47 \\
p=0.14\end{array}$ & $\begin{array}{l}0.93 \\
0.70\end{array}$ & $\begin{array}{l}0.53 \text { a } 1.64 \\
0.39 \text { a } 1.25\end{array}$ \\
\hline \multicolumn{6}{|l|}{ Horas de lazer por semana } \\
\hline $\begin{array}{l}0-2 \\
2 \text { a } 14 \\
>14 \\
\text { Parceiro fixo } \\
\text { Álcool } \\
\text { Tabagismo } \\
\text { Drogas ilícitas }\end{array}$ & $\begin{array}{l}26(29.5 \%) \\
50(56.82 \%) \\
12(13.64 \%) \\
53(60.22 \%) \\
47(53.41 \%) \\
9(10.23 \%) \\
19(21.6 \%)\end{array}$ & $\begin{array}{c}14(12.61 \%) \\
76(68.47 \%) \\
21(18.92 \%) \\
62(55.86 \%) \\
57(51.35 \%) \\
4(3.6 \%) \\
32(28.83 \%)\end{array}$ & $\begin{array}{l}\mathrm{p}<0.001 \\
\mathrm{p}=0.32 \\
\mathrm{p}=0.48 \\
\mathrm{p}=0.05 \\
\mathrm{p}=0.16\end{array}$ & $\begin{array}{c}\text { NA } \\
11.97 \\
1.09 \\
30.48 \\
0.68 \\
0.6798\end{array}$ & $\begin{array}{c}\text { NA } \\
0.67 \text { a } 2.1 \\
0.62 \text { a } 1.90 \\
0.90 \text { a } 10.25 \\
0.35 \text { a } 0.30\end{array}$ \\
\hline \multicolumn{6}{|l|}{ Série do Curso } \\
\hline $\begin{array}{l}1 \\
2 \\
3 \\
4 \\
5 \\
6 \\
\text { 1a-3aSéries } \\
4 a-6 a S e ́ r i e s \\
4 \\
5 \\
6\end{array}$ & $\begin{array}{l}17(19.32 \%) \\
21(23.86 \%) \\
10(11.36 \%) \\
12(13.64 \%) \\
12(13.64 \%) \\
16(18.18 \%) \\
48(54.55 \%) \\
40(45.45 \%) \\
12(13.64 \%) \\
12(13.64 \%) \\
16(18.18 \%)\end{array}$ & $\begin{array}{c}15(13.51 \%) \\
21(18.92 \%) \\
10(9.01 \%) \\
22(19.82 \%) \\
29(26.13 \%) \\
14(12.61 \%) \\
46(41.44 \%) \\
65(58.56 \%) \\
22(19.82 \%) \\
29(26.13 \%) \\
14(12.61 \%)\end{array}$ & $\begin{array}{l}\mathrm{p}=0.17^{*} \\
\mathrm{p}=0.04\end{array}$ & NA & $\begin{array}{c}\text { NA } \\
0.33 \text { a } 1.03\end{array}$ \\
\hline $\begin{array}{l}\text { Curso superior prévio } \\
\text { Falta de apoio emocional }\end{array}$ & $\begin{array}{c}24(27.27 \%) \\
66(75 \%)\end{array}$ & $\begin{array}{l}24(21.62 \%) \\
48(43.24 \%)\end{array}$ & $\begin{array}{l}p<0.001 \\
p=0.22 \\
p<0.001\end{array}$ & $\begin{array}{c}\text { NA } \\
0.6897 \\
0.254\end{array}$ & $\begin{array}{c}\text { NA } \\
0.35 \text { a } 1.3 \\
0.13 \text { a } 0.46\end{array}$ \\
\hline
\end{tabular}

* Teste $x 2$ unicaudado

\section{TABELA 6}

Médias e Desvios-Padrão do Escore de Depressão Beck (BDI) conforme a série do curso de Medicina

$\begin{array}{lc} & \begin{array}{c}\text { Escore de Beck } \\ \mathbf{n}=199 \\ \mathbf{m}(\mathbf{D P})\end{array} \\ \text { Sexta Série } & 9,73(5,39) \\ \text { Quinta Série } & 8,28(5,75) \\ \text { Quarta Série } & 9,5(6,76) \\ \text { Terceira Série } & 9,95(6,67) \\ \text { Segunda Série } & 11,88(7,21) \\ \text { Primeira Série } & 12,22(7,13)\end{array}$

Apenas a sexta série diferiu de modo estatisticamente significativo das demais turmas (Tukey-Kramer post-hoc analysis) $=>$ p=0,001 (teste de Levene)

\section{DISCUSSÃO}

A prevalência de sintomas depressivos entre estudantes de Medicina é uma constante em nível mundial, todavia seus valores apresentam um grande intervalo de variação, entre $13,9 \%$ e $79 \%{ }^{6-14}$. Prevalências de sintomas depressivos e de depressão severa entre acadêmicos de Medicina semelhantes às deste estudo foram encontradas numa universidade privada de Santa Catarina $(40,7 \%)^{6}$, numa universidade estadual no Paraná $(49,2 \%)^{7}$, na Arábia Saudita $(48,2 \%)^{10}$ e numa universidade de Hong Kong $(50 \%)^{14}$. Nesta última, os pontos de corte do BDI foram idênticos aos desta pesquisa e se constatou que, entre os estudantes de Medicina que apresentavam sintomas depressivos, $2 \%$ estavam severamente deprimidos ${ }^{14}$.

Resultados de outras universidades, que utilizaram os mesmos parâmetros do BDI, mostram a mesma tendência, na 
qual a prevalência dos transtornos depressivos leves é maior que a dos moderados e a dos graves, como ocorreu na Universidade de São Paulo, onde 24,9\% dos acadêmicos de Medicina tinham depressão leve, $11 \%$ moderada e 2,3\% severa9. Já em outro estudo, 19,9\% dos acadêmicos de Medicina apresentavam depressão leve, 5,9\% moderada e $1 \%$ grave $^{15}$.

$\mathrm{O}$ valor encontrado para as médias gerais dos escores do BDI neste estudo se enquadra nos padrões obtidos nos estudos comparados, que encontraram médias gerais para o BDI que variaram entre 5,77 e 10,7511,16,32,33.

Os prováveis agentes estressores presentes no curso de Medicina variam conforme a série e o período do curso (Tabela 5), o que foi demonstrado pela diferença estatisticamente significativa entre os dados obtidos no ciclo básico e no clínico. Porém, deve-se ressaltar que o valor de $p$ encontrado foi limítrofe e os cálculos não são fidedignos para que o ciclo clínico seja admitido como fator de risco para depressão pelo fato de a amostra da terceira série ter sido menor do que as demais. Entretanto, percebeu-se aumento progressivo da prevalência de depressão da quarta série em diante. A análise quantitativa dos escores do BDI entre todas as séries mostrou que apenas a sexta série diferiu de modo estatisticamente significativo das demais turmas, sugerindo que o fim do curso tende a ser o período mais exaustivo e que mais abala emocionalmente o acadêmico. Essa correlação significativa entre a variável quantitativa "pontuação obtida no BDI" e o final do curso também foi encontrada em Minas Gerais, indicando que, quanto mais elevada a série, maiores os valores de pontuação no $\mathrm{BDI}^{8}$.

Como apenas a sexta série diferiu de modo estatisticamente significativo em relação às demais quanto às médias do escore BDI, sugere-se que o final do curso de Medicina é o período que possui maior quantidade de fatores estressores e depressivos para o acadêmico. Outros estudos também relatam que o período de maior sofrimento psíquico vivenciado pelos estudantes corresponde àquele em que entram em contato com pacientes gravemente enfermos, o que significa, na realidade brasileira, os anos do internato, que inclui a última série do curso ${ }^{15,16,33}$. Alguns autores demonstram uma intensificação das queixas quanto a carga horária excessiva, falta de tempo para estudar e privação da vida social neste período ${ }^{20}$. Isto sugere que o currículo médico pode desempenhar um papel importante no aumento da prevalência de sintomas depressivos nos estudantes de Medicina, principalmente no final da graduação. Fatores como contato com a doença e a morte, agressividade inerente a muitas intervenções, dificuldades em comunicar más notícias, além da necessidade de especialização ao final do curso seriam responsáveis pelos sintomas depressivos mais intensos nesta população9. Contudo, em alguns estudos não foram encontradas diferenças significativas entre a sexta série e as outras séries do curso ${ }^{32,34}$.

A detecção precoce dos grupos de risco e a identificação das dificuldades experimentadas pelos alunos ao longo de cada etapa do curso podem ser indicativas da necessidade de desenvolver estratégias de enfrentamento e prevenção, por meio de projetos contínuos de psicoeducação que sensibilizem os estudantes para os riscos que transtornos psíquicos e disfunções profissionais são capazes de trazer para o seu bem-estar pessoal, de seus familiares e de seus pacientes ${ }^{26}$. Os programas psicoeducacionais são comprovadamente efetivos como terapia alternativa para a redução da prevalência de sintomas depressivos entre os estudantes de Medicina ${ }^{35}$

No presente estudo, por meio das múltiplas análises univariadas, observou-se que o estresse, a frequência de atividades de lazer, a satisfação com o desempenho acadêmico e a falta de apoio emocional no ambiente acadêmico são fatores de risco para o desenvolvimento de sintomas depressivos. Os acadêmicos que relataram gastar de zero a duas horas semanais em lazer e/ou atividades extracurriculares foram os que apresentaram maior escore no BDI, enquanto os que gastaram mais de 14 horas semanais nessas atividades foram os que mostraram menores pontuações. Em um estudo nordestino, a atividade de lazer também se mostrou significativamente correlacionada com depressão, pois os alunos de Medicina que esporadicamente e raramente tinham atividades de lazer se apresentaram mais vulneráveis a sintomas depressivos ${ }^{17}$.

O estresse como fator associado à depressão tem chamado a atenção nos estudantes de Medicina ${ }^{18}$. Um estudo realizado na Arábia Saudita demonstrou uma alta carga de estresse durante o curso, o que tem implicações no rendimento acadêmico, uma vez que pode acarretar e/ou piorar distúrbios mentais, impactando negativamente a função cognitiva e o aprendizado $^{19}$. Na UEPG, a quase totalidade dos acadêmicos de Medicina pesquisados admitiu ser estressada, e a maior parte deles considera elevado seu grau de estresse. Abdulghani $^{19}$ descreve que $57 \%$ dos acadêmicos de Medicina de sua universidade assumiram ser estressados, tendo 19,6\% deles alto nível de estresse. Essas importantes prevalências relacionadas ao nível de estresse indicam que o curso de Medicina é por si só um fator estressor para o acadêmico e que esse estresse é um fator de risco para o aparecimento de sintomas depressivos ${ }^{19,36}$.

O estado civil, o uso de álcool e de drogas ilícitas e o sexo não apresentaram significância estatística neste estudo, o que se confirma em outros estudos, que também não demonstraram relevância para depressão em estudantes de Medicina ${ }^{19,17,37}$. 
Este trabalho apresentou menor participação do sexo feminino. Mesmo assim, não se identificou diferença estatística significativa quanto à presença de depressão entre os estudantes em relação ao sexo, assim como num estudo realizado em Sorocaba $^{32}$. Porém, vale ressaltar que existe na literatura maior prevalência de sintomas depressivos em mulheres, tanto no meio acadêmico como na população geral ${ }^{6,7,17}$. Em relação ao tabaco, estudos têm demonstrado maiores chances de desenvolvimento de sintomas depressivos em seus usuários.

Millan e Arruda ${ }^{38}$ relataram que os estudantes de Medicina que apresentam melhor desempenho escolar são os mais exigentes e, consequentemente, estão mais propensos a desenvolver sintomas depressivos e a sofrer as pressões impostas ante qualquer falha. Outro estudo também correlacionou o desempenho acadêmico dos alunos de Medicina com sintomas depressivos e encontrou significância estatística ${ }^{16}$, o que sustenta o resultado encontrado na presente pesquisa. A falta de apoio emocional pode estar envolvida na gênese do transtorno depressivo, pois pode estar relacionada com a abordagem inadequada do sintoma depressivo, deixando o indivíduo mais vulnerável perante suas percepções das atividades cotidianas.

Figura 1

Prevalência de utilização de antidepressivos

$(n=36)$ e suas respectivas classes dentre os

acadêmicos do curso de Medicina da Universidade

Estadual de Ponta Grossa durante o período de setembro de 2014 a fevereiro de 2015

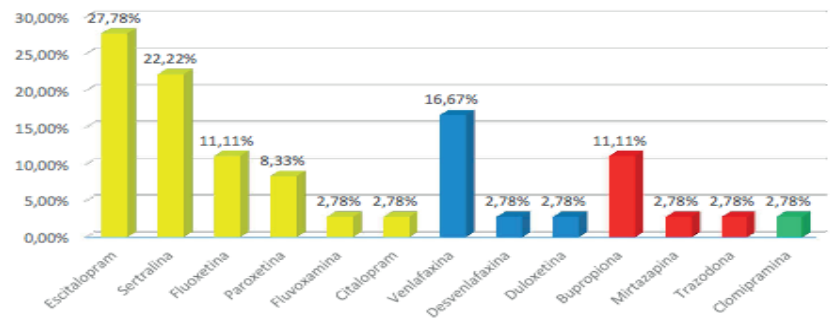

Estima-se que o uso de medicamentos antidepressivos por jovens chegue a $8,3 \%{ }^{39}$. De acordo com os resultados deste trabalho, identificou-se que esse número pode ser maior em acadêmicos de Medicina, uma vez que 29,15\% dos acadêmicos pesquisados utilizam ou já utilizaram fármacos antidepressivos e 18,09\% estavam administrando esses medicamentos no momento da pesquisa, sendo o Escitalopram o medicamento mais prevalente (Figura 1). Uma faculdade pública de Medicina em São Paulo relata que $11,4 \%$ de seus acadêmicos utilizam ou já utilizaram fármacos antidepressivos, sendo a Fluoxetina o medicamento mais utilizado $(33,3 \%)^{20}$. Os dados, portanto, são validados pela literatura, que define os ISRS como os antidepressivos mais prescritos atualmente ${ }^{4}$.

É relevante, no presente estudo, o fato de que apenas $33,3 \%$ dos usuários de medicamentos antidepressivos possuem alto grau de adesão ao tratamento. Isto é de especial importância porque a baixa adesão ao tratamento pode levar ao agravamento da doença e à redução da capacidade cognitiva. No caso da depressão, a não adesão ao tratamento também pode trazer consequências letais, considerando o alto risco de suicídio nas pessoas que apresentam distúrbios depressivos ${ }^{40}$.

A análise deste contexto sugere que acadêmicos de Medicina são relutantes quanto à procura de tratamento adequado, por provável medo do estigma, pela questão da confidencialidade ou por dificuldades financeiras ${ }^{15}$. Nesta investigação, isso parece se confirmar, já que apenas um pequeno percentual tem adesão elevada ao tratamento medicamentoso, apesar da prevalência encontrada para os sintomas depressivos e do fato de $77,8 \%$ dos usuários de medicamentos antidepressivos dessa amostra apresentarem escore de Beck $\geq 11$ pontos, o que sugere que o transtorno depressivo está sendo tratado inadequadamente.

A grande variação da prevalência de depressão parece indicar vieses nas coletas de dados e na interpretação do BDI, o que dificulta a realização de comparações entre os estudos, permitindo apenas considerá-los semelhantes. Foi detectado, no presente estudo, que houve viés de amostragem na terceira série do curso, uma vez que apenas 51\% da turma foram submetidos à pesquisa. Salienta-se que todos os estudos analisa$\operatorname{dos}^{6-14}$ que relacionam depressão com estudantes de Medicina indicam prevalência maior de depressão neste grupo do que na população geral ${ }^{41}$.

\section{CONCLUSÃO}

As prevalências de sintomas depressivos nos acadêmicos de Medicina da UEPG e da utilização de medicamentos antidepressivos vão ao encontro dos dados referentes a acadêmicos de Medicina de outras instituições brasileiras e internacionais ${ }^{3,41,42}$. Apresentam-se como fatores de risco para os transtornos depressivos a frequência das atividades de lazer, o estresse, a satisfação com o desempenho acadêmico e a falta de apoio emocional no ambiente acadêmico. A prevalência de depressão a partir da quarta série tendeu a aumentar, e apenas a sexta série diferiu de modo estatisticamente significativo em relação às demais quanto às médias do escore BDI, o que sugere a hipótese de que o final do curso de Medicina é o período que apresenta maior quantidade de fatores estressores e depressivos para o acadêmico. 


\section{REFERÊNCIAS}

1. Üstün TB, Kessler RC. Global burden of depressive disorders:the issue of duration. Br J Psychiatry.2002; 181(3):181-3.

2. Chen YW, Dilsaver SC. Lifetime rates of suicide attempts among subjects with bipolar and unipolar disorders relative to other Axis I disorders. Biol Psychiatry.1996; 39(10):896-9.

3. Del Porto JA. Conceito e diagnóstico de depressão. Rev Bras Psiquiatr.1999; 21 (1):S6-10.

4. O'Donnell JM, Shelton RC. Tratamento farmacológico da depressão e dos transtornos de ansiedade. In : Brunton LL, org; Chabner BA, org; Knollmann BC, org.As bases farmacológicas da terapêutica de Goodman \& Gilman. 12.ed. Porto Alegre:AMGH; 2012. p. 397-417.

5. Suominen KH, Isometa ET, Henriksson MM, Ostamo AI,Lönnqvist JK.Inadequate treatment for major depression both before and after attempted suicide. Am J Psychiatry.1998; 155 (12):1778-80.

6. Moro A, Vale J, Lima L. Sintomas Depressivos nos Estudantes de Medicina da Universidade da Região de Joinville (SC). Rev Bras Educ Med.2005; 29(2):97-102.

7. Porcu M, Fritzen VC, Helber C. Sintomas depressivos nos estudantes de Medicina da Universidade Estadual de Maringá. Psiquiatr Prat Médica [Internet]. 2001.34(1) [capturado 19 abr. 2014]. Disponível em: http://www.unifesp. $\mathrm{br} / \mathrm{dpsiq} / \mathrm{polbr} / \mathrm{ppur} /$ original501.htm.

8. Abrao CB, Coelho ED, Passos LBS. Prevalência de sintomas depressivos entre estudantes de medicina da Universidade Federal de Uberlândia. Rev Bras Educ Med.2008; 32(3):315-23.

9. Baldassin S, Alves TC, de Andrade AG, Nogueira Martins LA. The characteristics of depressive symptoms in medical students during medical education and training: a cross-sectional study. BMC Med Educ [Internet].2008.11[capturado 19 abr. 2014]; 8:60.

10. Al-Faris EA, Irfan F, Van der Vleuten CP, Naeem N, Alsalem A, Alamiri $\mathrm{N}$ et al. The prevalence and correlates of depressive symptoms from an Arabian setting: a wakeup call. Med Teach.2012; 34 (1):S32-36.

11. Ahmed I, Banu H, Al-Fageer R, Al-Suwaidi R. Cognitive emotions: depression and anxiety in medical students and staff. J Crit Care.2009.24(3):1-7.

12. Givens JL, Tjia J. Depressed medical students' use of mental health services and barriers to use. Acad Med.2002; 77(9):918-21.

13. Curran TA, Gawley E, Casey P, Gill M, Crumlish. Depression, suicidality and alcohol abuse among medical and business students. Ir Med J.2009;102(8):249-52.
14. Chan DW. Depressive symptoms and depressed mood among Chinese medical students in Hong Kong. Compr Psychiatry.1991; 32(2):170-80.

15. Amaral GR, de Paula LMG, Batista MP, Píccolo PP, Teles TBG, Oliveira PM,et al. Sintomas depressivos em acadêmicos de medicina da Universidade Federal de Goiás: um estudo de prevalência. Rev Psiquiatr.RS. 2008; 30(2):124-30

16. Costa EFO, Santana YF, Santos ATRA, Martins LAN, De Melo EV, Andrade TM. Sintomas depressivos entre internos de medicina em uma universidade pública brasileira. Rev Assoc Med Bras.2012; 58(1):53-9.

17. De Paula JA, Borges AMFS, Bezerra LRA, Parente HV, De Paula RCA, Wajnsztejn R,et al. Prevalência e fatores associados à depressão em estudantes de Medicina. Journal of Human Growth and Development. 2014; 24(3):274-81.

18. Dahlin M, Joneborg N, Runeson B. Stress and depression among medical students: a cross-sectional study. Med Educ.2005; 39(6):594-604.

19. Abdulghani HM.Stress and depression among medical students: a cross sectional study at a medical college in Saudi Arabia.Pak J Med Sci.2008;24(1):12-17.

20. 20.Ribeiro AG, da Cruz LP, Marchi KC, Tirapelli CR, Miasso AI. Antidepressivos: uso, adesão e conhecimento entre estudantes de medicina. Ciênc saúde coletiva. 2014;19(6):1825-33.

21. Nadal-Vicens M, Chyung JH, Turner TJ. Farmacologia da Neurotransmissão Serotoninérgica e Adrenérgica Central. In. Golan E et al. Princípios de Farmacologia: As Bases Fisiopatológicas da Farmacoterapia. 2.ed.São Paulo:Guanabara Koogan;2009 . p .187-202.

22. Duman RS. Pathophysiology of depression and innovative treatments: remodeling glutamatergic synaptic connections. Dialogues in Clin Neurosci.2014; 16(1):11-27.

23. Wang YP, Gorenstein C. Assessment of depression in medical patients: a systematic review of the utility of the Beck Depression Inventory- II. Clinics. 2013; 68(9):1274-87.

24. Gorenstein C, Andrade L. Validation of a Portuguese version of the Beck Depression Inventory and the State-Trait Anxiety Inventory in Brazilian subjects. Braz Med Biol Res.1996;29(4):453-7.

25. Gomes-Oliveira MH, Gorenstein C, Neto FL, Andrade LH, Wang YP. Validation of the Brazilian Portuguese version of the Beck Depression Inventory-II in a community sample. Rev Bras Psiquiatr.2012; 34(4):389-94.

26. Ben AJ, Neumann, RC, Mengue SS. Teste de Morisky-Green e Brief Medication Questionnaire para avaliar adesão a medicamentos. Rev Saúde Pública.2012; 46(2):279-89. 
27. Morisky DE, Levine M, Green LW, Smith CR. Health education program effects on the management of hypertension in the elderly. Arc. Intern Med.1982; 142(10):1835-38.

28. Morisky DE, Green LW, Levine DM. Concurrent and predictive validity of self-reported measure of medication adherence. Med Care.1986; 24(1): 67-74.

29. 29.Beck AT, Steer RA, Brown GK. Manual for the Beck Depression Inventory - Fast Screen for Medical Patients.San Antonio. TX:Psychological Corporation; 2000.

30. Beck AT, Ward CH, Mendelson M, Mosk J, Erbaugh I.An inventory for measuring depression. Arch Gen Psychiatry. $1961 ; 4: 561-71$.

31. Schafranski MD. O Mínimo:14 breves capítulos para entender os fundamentos da Bioestatística. Ponta Grossa: UEPG; 2014.

32. Vallilo NG, Júnior RD, Gobbo R, Novo NF, Hübner CVK. Prevalência de sintomas depressivos em estudantes de Medicina. Rev Bras Clin Med.2011.9(1):36-41.

33. Goebert D, Thompson D, Takeshita K, Beach C, Bryson P, Ephgrave $\mathrm{K}$ et al. Depressive symptoms in medical students and residents: a multischool study. Acad Med.2009; 84(2):236-41.

34. Bassols AM, Okabayashi LS,Silva AB,Carneiro BB,Feijó F,Guimarães GC et al. First and last year medical students: is there a difference in the prevalence and intensity of anxiety and depressive symptoms?Rev Bras Psiquiatr.2014; 36(3):233-240.

35. Melo-Carrillo A, Oudenhove LV, Lopez-Avila A. Depressive symptoms among Mexican medical students: High prevalence and the effect of a grouppsychoeducation intervention. J Affect Disord. 2012; 136(3):1098-103.

36. Dyrbye LN, Thomas MR, Shanafelt TD. Systematic Review of Depression, Anxiety, and Other Indicators of Psychological Distress Among U.S. and Canadian Medical Students. Academic Medicine. 2006; 81(4):354-73.

37. Jadoon NA, Yagoob R, Raza A, Shehzad MA, Zeshan SC. Anxiety and depression among medical students: a cross-sectional study. J Pak Med Assoc.2010; 60(8): 699-702.

38. Millan LR, Arruda PC. Assistência psicológica ao estudante de medicina: 21 anos de experiência. Rev Assoc Med Bras.2008; 54(1):90-4.
39. 39. Adewuia AO, Ola BA, Aloba OO, Mapayi BM, Oginni OO. Depression amongst Nigerian university students: prevalence and sociodemographic correlates. Soc Psychiatr Epidemiol [Internet].2006.41(8)[capturado 28abr.2014]; 674-8.http: / / link.springer.com/article / 10.1007\%2 Fs00127-006-0068-9

40. Oliê JP, Silva, JAC, Macher JP. Neuroplasticity and a new approach to the pathophysiology of depression. London: Current Medicine Groups; 2005.

41. Cunha RV, Bastos GAN, Del Duca GF. Prevalência de depressão e fatores associados em comunidade de baixa renda de Porto Alegre, Rio Grande do Sul. Rev Bras Epidemiol.2012;15(2):346-54.

42. Güleç M, Bakir B, Ozer M, Uçar M, Kiliç S, Hasde M. Association between cigarette smoking and depressive symptoms among military medical students in Turkey. Psychiatry Res.[Internet] 2005.134(3)[capturado 28abr.2014];281-6. http://www.psy-journal.com/article/ S0165-1781(05)00058-2/abstract

\section{CONTRIBUIÇÃO DOS AUTORES}

Este trabalho foi desenvolvido através de projeto de pesquisa sob a orientação do autor principal, Fabiana Postiglione Mansani; a coleta de dados e as análises estatísticas foram realizadas pela acadêmica do Curso de Medicina Cynthia Ajus Cybulski.

\section{CONFLITO DE INTERESSES}

Não existem conflito de interesses por parte dos autores.

\section{ENDEREÇO PARA CORRESPONDÊNCIA}

Fabiana Postiglione Mansani

Universidade Estadual de Ponta Grossa

Av. Carlos Cavalcanti, 4748

Uvaranas - Ponta Grossa

CEP 84030-900 - PR

E-mail: cynthiacybulski@hotmail.com; fmansani@uepg.br 